\title{
Minor influence of humeral component size on torsional stiffness of the Souter-Strathclyde total elbow prosthesis
}

\author{
Joris C. T. van der Lugt • Daniel R. Suarez • \\ Tim J. van der Steenhoven • Rob G. H. H. Nelissen
}

Received: 31 August 2009 /Revised: 28 October 2009 / Accepted: 1 November 2009 / Published online: 1 December 2009

(C) The Author(s) 2009. This article is published with open access at Springerlink.com

\begin{abstract}
The use of Souter-Strathclyde total elbow prostheses is a well-studied replacement therapy for reconstruction of the elbow, but loosening of the humeral component is still of concern at long-term follow-up. In this study we looked at the effect of humeral component size and bone mineral density with respect to the bone size, torsional stiffness and torque to failure in cadaveric bones. Fourteen cadaveric humeri were available for testing purposes and four different humeral component size categories were used. First, we calculated the bone quality using dualenergy X-ray absorptiometry (DEXA). The torsional stiffness of the prosthetic humeri was measured during two mechanical tests: Firstly, the applied torque was recorded during a torsion fatigue test. The change of torsional stiffness between the tenth and last cycle was calculated. Secondly, a simple torsion test was performed and the torque to failure was recorded. No significant differences in outcome were seen between sizes of humeral components, even after correction for the bone size. Torsional stiffness and torque to failure were significantly correlated with bone mineral density and not with component size. In conclusion, bone quality seems to be a major eminent factor in the fixation of the humeral component in elbow replacement surgery.
\end{abstract}

J. C. T. van der Lugt $(\bowtie) \cdot$ D. R. Suarez $\cdot$

T. J. van der Steenhoven - R. G. H. H. Nelissen

Department of Orthopaedic Surgery,

Leiden University Medical Center (LUMC),

P.O. Box 9600, 2300 RC Leiden, The Netherlands

e-mail: j.c.t.van_der_lugt@lumc.nl

\section{Introduction}

The use of Souter-Strathclyde total elbow prostheses is a wellstudied replacement therapy for reconstruction of the elbow joint, especially in rheumatoid arthritis (Fig. 1) [1-4]. The overall survival rate of this cemented prosthesis is $77.4 \%$ after ten years, and this rate is comparable with other elbow prostheses. Aseptic loosening of the humeral component is the most common reason for failure of this prosthesis [5]. Due to their better long-term results, long-stem humeral components are now advocated by some authors [1-3]. However, the disadvantage of these long-stem components is the increased difficulty in revision surgery caused by decreased bone stock and subsequent increased risk of an intraoperative fracture.

In daily activities lifting movements with a flexed elbow are very common, for example when drinking, combing hair and facial washing. These movements cause an internal rotational loading which is directed on to the humeral component. The Souter-Strathclyde total elbow prosthesis is a non-constrained prosthesis, but a biomechanical study using cadaveric elbows showed that it is more constrained than other non-constrained types, which further increases the rotational loading on the humeral component [6]. As seen in previous studies, the loosening process starts at the condyles and is followed by an anterior tilting of the humeral component [7, 8]. For this reason, the designer of the prosthesis (W.A. Souter) advised that the condyles be excavated as much as possible to insert a relatively large component [9]. Removal of much cancellous bone at the condyles should provide a more rigid cortical fixation of the component during rotational loadings, but this theory has not yet been proven. In this cadaveric study, we looked at the effect of humeral component size, stem length and relation to the bone size, on torsional stiffness and torque to failure in cadaveric bones. 
Fig. 1 Three standard humeral components (sized small, medium and large) and one medium long-stem humeral component of the SouterStrathclyde total elbow prosthesis

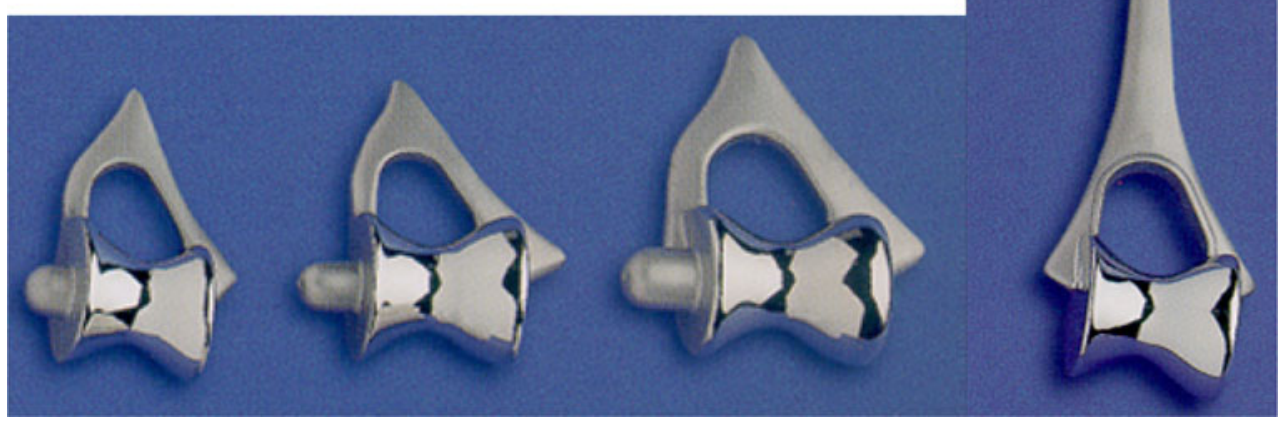

\section{Material and methods}

Fourteen human cadaveric humeri from donors were available for testing purposes (Table 1). The first goal of the study was to determine the difference in stiffness and torque to failure with respect to component size in relation to bone size. The humeral component of the SouterStrathclyde prosthesis is made of Vitallium and has a flat intramedullary stem. The component sizes which can be routinely used during surgery are small, medium and large.
The study components were formerly loosened prostheses, which were revised and thus available for this study. We used five standard small components, two standard medium components, one standard large component and six longstem medium components for insertion.

The second goal was to determine the difference in stiffness and torque to failure between standard components (eight) and long-stem components (six); the latter component is only available in one size (medium) at our centre.

Table 1 Characteristics of the 14 elbows with comparison of the means for the standard (size: S, M or L) and the long-stem humeral component (size: LS) groups using Student's $t$ test. Initial and final stiffness refer to torsional stiffness at the tenth and last cycles, respectively

\begin{tabular}{|c|c|c|c|c|c|c|c|c|c|c|}
\hline Elbow & Age & Sex & Side & Size & Ratio & $\begin{array}{l}\mathrm{BMD} \\
\left(\mathrm{g} / \mathrm{cm}^{2}\right)\end{array}$ & T-score & $\begin{array}{l}\text { Initial stiffness } \\
\left(\mathrm{Nm} /{ }^{\circ}\right)\end{array}$ & $\begin{array}{l}\text { Final stiffness } \\
\left(\mathrm{Nm} /{ }^{\circ}\right)\end{array}$ & $\begin{array}{l}\text { Torque to } \\
\text { failure }(\mathrm{Nm})\end{array}$ \\
\hline 1 & 98 & M & $\mathrm{L}$ & $\mathrm{S}$ & 0.52 & 0.777 & -1.7 & 6.09 & 4.83 & 29.62 \\
\hline 2 & 67 & $\mathrm{M}$ & $\mathrm{L}$ & $\mathrm{S}$ & 0.52 & 0.957 & -0.5 & 6.08 & 6.41 & 36.72 \\
\hline 3 & 75 & $\mathrm{M}$ & $\mathrm{R}$ & $\mathrm{S}$ & 0.52 & 1.196 & 1.1 & 3.56 & 7.65 & 79.33 \\
\hline 4 & 84 & M & $\mathrm{L}$ & $\mathrm{S}$ & 0.53 & 0.881 & -0.5 & 9.88 & 9.27 & 65.41 \\
\hline 5 & 80 & $\mathrm{~F}$ & $\mathrm{R}$ & $\mathrm{S}$ & 0.63 & 0.495 & -3.7 & 2.33 & 2.38 & 21.55 \\
\hline 6 & 84 & F & $\mathrm{R}$ & M & 0.75 & 0.439 & -4.1 & 6.29 & 6.02 & 21.57 \\
\hline 7 & 93 & $\mathrm{~F}$ & $\mathrm{R}$ & M & 0.76 & 0.543 & -3.3 & 3.81 & 2.17 & 23.18 \\
\hline 8 & 62 & M & $\mathrm{R}$ & $\mathrm{L}$ & 0.82 & 0.814 & -1.4 & 6.21 & 5.77 & 31.01 \\
\hline 9 & 84 & $\mathrm{M}$ & $\mathrm{R}$ & LS & 0.54 & 0.844 & -0.8 & 3.61 & 3.63 & 39.24 \\
\hline 10 & 85 & $\mathrm{~F}$ & $\mathrm{R}$ & LS & 0.58 & 0.338 & -4.9 & 3.05 & 3.22 & 27.60 \\
\hline 11 & 85 & F & $\mathrm{L}$ & LS & 0.59 & 0.340 & -4.9 & 3.55 & 2.89 & 16.48 \\
\hline 12 & 82 & $\mathrm{~F}$ & $\mathrm{~L}$ & LS & 0.62 & 0.715 & -1.9 & 5.73 & 4.73 & 32.21 \\
\hline 13 & 93 & $\mathrm{~F}$ & $\mathrm{~L}$ & LS & 0.63 & 0.502 & -3.6 & 4.67 & 3.53 & $\mathrm{a}$ \\
\hline 14 & 80 & $\mathrm{~F}$ & $\mathrm{~L}$ & LS & 0.63 & 0.472 & -3.9 & 3.72 & 3.10 & 25.93 \\
\hline Mean total group (SE) & $82(2.6)$ & & & & $0.62(0.026)$ & $0.665(0.0685)$ & $-2.4(0.50)$ & $4.90(0.523)$ & $4.69(0.563)$ & $34.60(5.027)$ \\
\hline $\begin{array}{l}\text { Mean standard group } \\
(\mathrm{SE})(N=8)\end{array}$ & $80(4.3)$ & & & & $0.63(0.045)$ & $0.763(0.0913)$ & $-1.8(0.64)$ & $5.53(0.819)$ & $5.56(0.858)$ & $38.5(7.72)$ \\
\hline $\begin{array}{l}\text { Mean long-stem group } \\
\text { (SE) }(N=6)\end{array}$ & $85(1.8)$ & & & & $0.59(0.014)$ & $0.535(0.0836)$ & $-3.3(0.68)$ & $4.06(0.398)$ & $3.52(0.267)$ & $28.3(3.75)$ \\
\hline Student's $t$ test & $p=0.37$ & & & & $p=0.51$ & $p=0.09$ & $p=0.12$ & $p=0.14$ & $p=0.05$ & $p=0.26$ \\
\hline
\end{tabular}

$B M D$ bone mineral density, $S E$ standard error of the mean

${ }^{a}$ No measurement 
The maximum distance between the condyles of each cadaveric humerus was measured with a digital marking gauge. The same procedure was performed for the maximum distance between the condyles of the humeral component. Bones and their corresponding components were chosen by the first author (JvdL) in order to have, as much as possible, different component to bone ratios (defined as the ratio between the bone width and the implant width). Randomisation was not possible, because the large components did not fit into all humeri. A high ratio means that more excavation of the condyles was needed to insert a relatively large component.

All humeri were obtained from the Department of Anatomy and Embryology, Leiden University Medical Center (LUMC). No approval of the Ethics Committee was needed. Fixation and preservation of all cadavers were performed by injection of embalming fluid into the femoral artery, consisting of $36 \%$ formaldehyde with a mixture of ethanol, glycerine, phenol, $\mathrm{K}_{2} \mathrm{SO}_{4}, \mathrm{Na}_{2} \mathrm{SO}_{4}, \mathrm{NaHCO}_{3}$, $\mathrm{NaNO}_{3}$ and $\mathrm{NaSO}_{3}$.

The degree of osteoporosis of each proximal humerus was determined using dual-energy X-ray absorptiometry (DEXA) with a Discovery A, QRD scanner (Hologic Inc., Bedford, MA, USA). Osteopaenia and osteoporosis were defined using T-scores of $<-1$ standard deviation and $<-2.5$ standard deviation from the young adult mean value, respectively.

All components were fixed with Biomet Bone Cement (Biomet b.v., Dordrecht, The Netherlands) after rinsing the bone surfaces with water until the bone trabeculae were visible. No cement stops were used, and the humeral canal was occluded with bone debris. All prostheses were inserted by the senior author (RGN) (Fig. 2). Standard radiographs in two directions were made before testing to exclude complications such as inadequate cement mantle (i.e. mantle defects or a mantle with less than $1 \mathrm{~mm}$ thickness), cortical perforation, fractures or incorrect prosthetic position. No complications were seen on these conventional radiographs in two directions (Fig. 3).

A custom-made testing device was designed and built to accomplish this study (Fig. 4). It enabled a rigid fixation of the proximal humeral bone and the application of a specific angular deformation at the prosthetic elbow joint during the different tests. The loading was applied by a testing machine (LR5KPlus, XLC-50K-A1, Lloyd Instruments, Fareham, Hants, UK). The displacement applied by this testing machine to the custom-made testing device and the reaction force to it were recorded using NEXYGENPlus software (Lloyd Instruments, Fareham, Hants, UK). Thereafter, the angular deformation of, and the torque applied to, the specimens were calculated. The torque was corrected for the effect of the angular deformation.

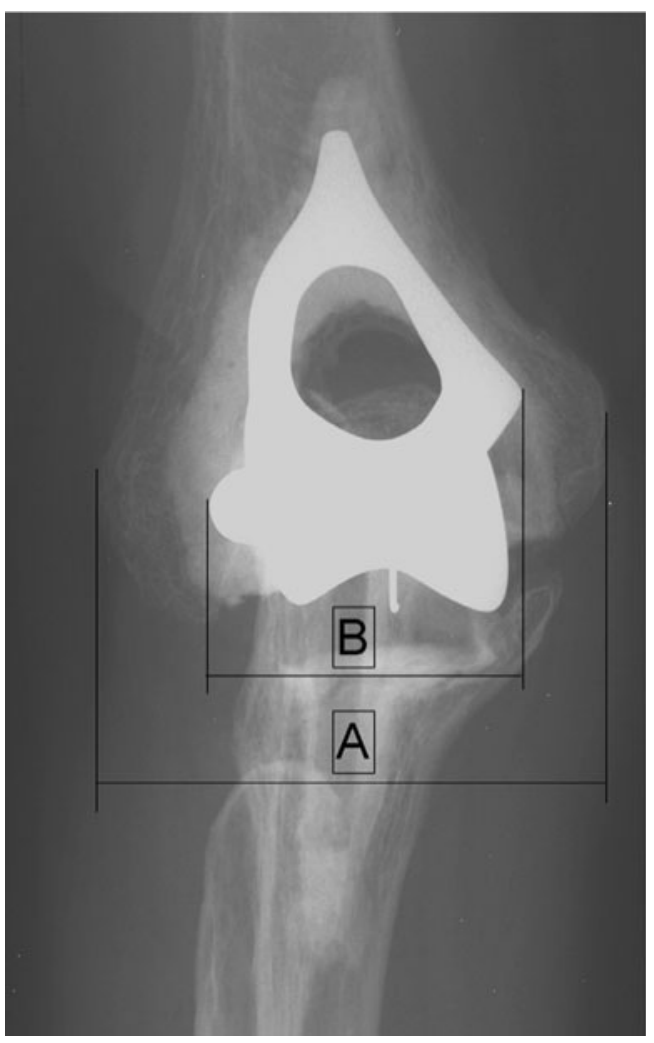

Fig. 2 The ratio between the maximal width of the component (b) and the maximal width of the bone (a)

In contrast to a previous biomechanical study with continuous loading at our centre, the specimens were tested first using a fatigue test to simulate a realistic clinical situation in order to determine how the implant fixation would change during daily use (initial torsional stiffness) [10]. Thereafter, a single torsion test until fracture was used, and the torque to failure of the bone

Fig. 3 All components were inserted using a tamp

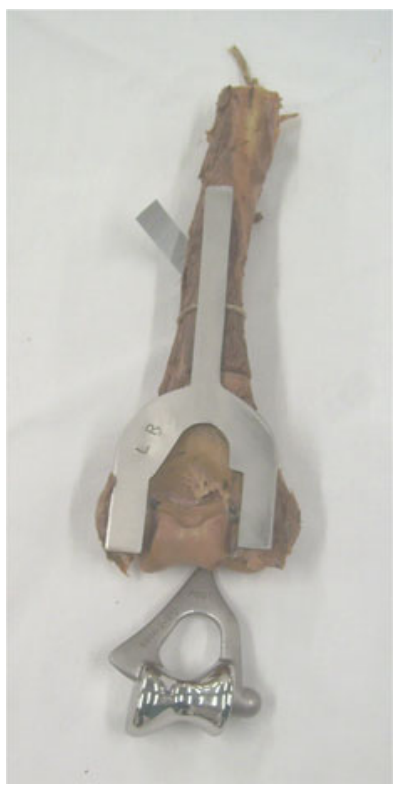


Fig. 4 The testing device

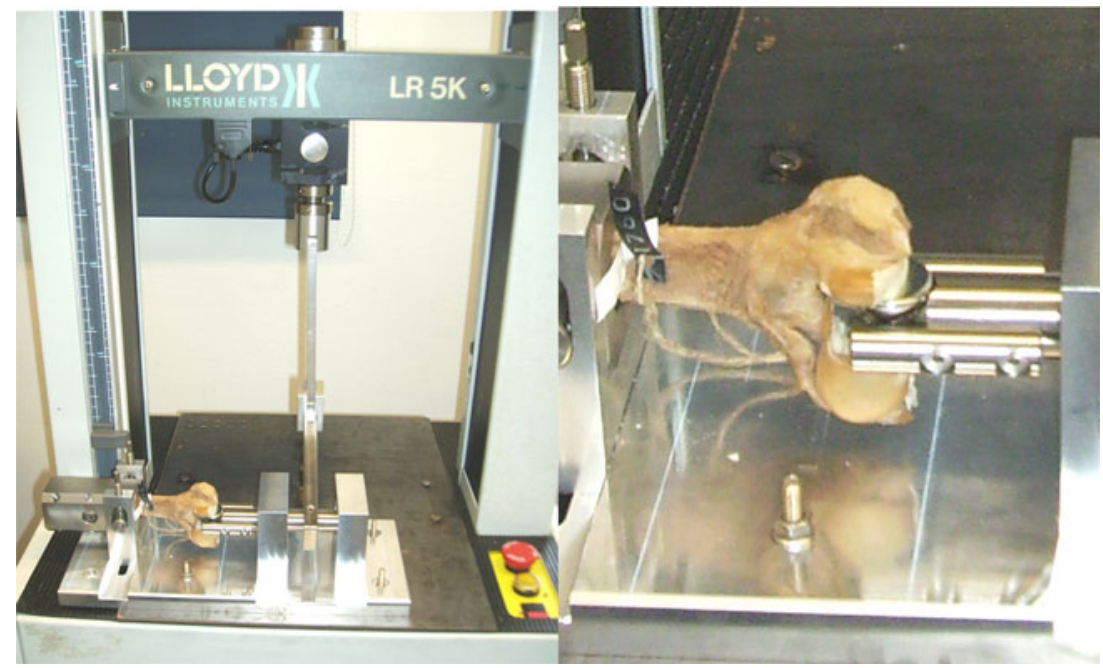

(i.e. fracture) was measured [10]. In the fatigue test the specimens were loaded with cyclic axial torsion (twist angle between 0 and $4^{\circ}$, elbow's internal rotation) during 200 cycles. This number of cycles estimates the change of torsional stiffness between immediately after surgery and some weeks later. An elastic behaviour of the bone, without tendency to fracture, was seen in preliminary tests when the angular deformation was kept in this range of 200 cycles of $0-3.8^{\circ}$. Change in the torsional stiffness was calculated between the tenth and the last cycles. We induced axial prestrains in the sample when the sample was fixed in the gripper which gives the torque. That prestrain makes the construction stiffer than it really is. In the single torsion tests the specimens were loaded with internal rotation until fracture. The moment arm was $15 \mathrm{~cm}$ and had a linear velocity of $10 \mathrm{~mm} / \mathrm{s}$ which gave an angular velocity of $0.06 \mathrm{rad} / \mathrm{s}=3.8 \% \mathrm{~s}$ ). The speed for the single torsion test was $50 \mathrm{~mm} / \mathrm{min}$.

The experimental set-up and protocol were determined arbitrarily, since for elbow prostheses no literature with similar tests was available. Only some biomechanical cadaveric studies on plate or nail fixation for humeral fractures are available. Blum et al. measured bending and torsional properties of humeral intramedullary nails at 4 , 6 and $10 \mathrm{Nm}$ with an angular deformation of $30^{\circ}$ [11]. Stoffel et al. used $2 \mathrm{Nm}$ at $0.5 \%$ during 20 cycles for calculation of the stability after humeral plating [12]. In our study we had from 9 up to $40 \mathrm{Nm}$ with an angular deformation of $4^{\circ}$ during the cyclic loading tests.

\section{Statistical analysis}

Student's $t$ test was applied to compare the standard and long-stem component groups. Pearson's correlation test was used to investigate the effect of component to bone ratios and bone densities on loading tests. A regression analysis with curve estimation was performed to show the relation between ratio and bone mineral density (BMD) on stiffness and maximal torque. For all tests the level of

Table 2 Pearson's correlation test for the 14 elbows (significance at $p<0.05$ ). Significance marked as bold numbers. Initial and final stiffness refer to torsional stiffness at the tenth and last cycles, respectively

\begin{tabular}{|c|c|c|c|c|c|c|c|}
\hline Correlations & Age & Size component & Ratio & BMD & Initial stiffness & Final stiffness & Torque to failure \\
\hline Age & 1 & $0.10(p=0.73)$ & $-0.17(p=0.56)$ & $-0.41(p=0.14)$ & $-0.09(p=0.76)$ & $-0.37(p=0.19)$ & $-0.23(p=0.46)$ \\
\hline Size component & & 1 & $0.19(p=0.51)$ & $-0.51(p=0.06)$ & $-0.34(p=0.23)$ & $-0.52(p=0.06)$ & $-0.43(p=0.16)$ \\
\hline Ratio & & & 1 & $-0.39(p=0.17)$ & $-0.03(p=0.91)$ & $-0.27(p=0.36)$ & $-0.49(p=0.09)$ \\
\hline BMD & & & & 1 & $0.37(p=0.19)$ & $0.71(p<0.01)$ & $0.83(p<0.01)$ \\
\hline Initial stiffness & & & & & 1 & $0.78(p<0.01)$ & $0.35(p=0.25)$ \\
\hline Final stiffness & & & & & & 1 & $0.78(p<0.01)$ \\
\hline Torque to failure & & & & & & & 1 \\
\hline
\end{tabular}

$B M D$ bone mineral density 
significance was set at $p<0.05$. All data were analysed with SPSS version 17.0 (SPSS Inc., Chicago, IL, USA).

\section{Results}

No prostheses loosened completely during the cyclic loading. All elbows fractured during the single torsion test, and in all cases the fracture line ended at the proximal tip of the component. As a consequence more bone stock was maintained after the fracture with the relatively smaller humeral components. During the fatigue tests increasing micromotions of up to a few millimetres were seen in all cases between the interface of cement and bone. These bone-cement interface micromotions originated at the humeral condyle level and propagated proximally to the tip of the prosthesis.

No significant differences in outcome were seen between the standard $(N=8)$ and long-stem component groups $(N=6)$, but a trend was seen for increased final torsional stiffness in the standard component group $(p=0.05$, Table 1$)$.

Initial and final torsional stiffness and torque to failure were not significantly correlated to the component to bone ratio or to the component size. In addition, final stiffness and torque to failure showed a positive correlation to BMD (Table 2). Regression analysis showed a significant influence of BMD on final stiffness and the torque to failure ( $r=0.71, p=<0.01$ and $r=0.83, p<0.01$, respectively, Fig. 5).

\section{Discussion}

In this cadaveric study we looked at the effect of the humeral component size of the Souter elbow prosthesis and humeral component stem length on torsional stiffness and torque to failure in cadaveric bones. As advocated by Souter, removal of most cancellous bone at the condyles should provide a more rigid cortical fixation of the humeral component during rotational loadings of the elbow, but this theory has not yet been proven.

In contrast to the usual clinical situation, these cadaveric bones were not affected by rheumatoid arthritis. The BMD is often not determined in general practice, but most rheumatoid patients have some degree of osteoporosis due to rheumatoid medication, mainly in the peripheral bones [13]. We used cadaveric bones with a wide range of BMD. The influence of BMD on the mechanical failure of the prosthesis-cement-bone interface in this study was obvious, suggesting that the influence of bone quality on prosthetic fixation should be the aim for research in elbow replacement surgery.

The preference for using long-stem components in the recent literature could not be supported by this biome-
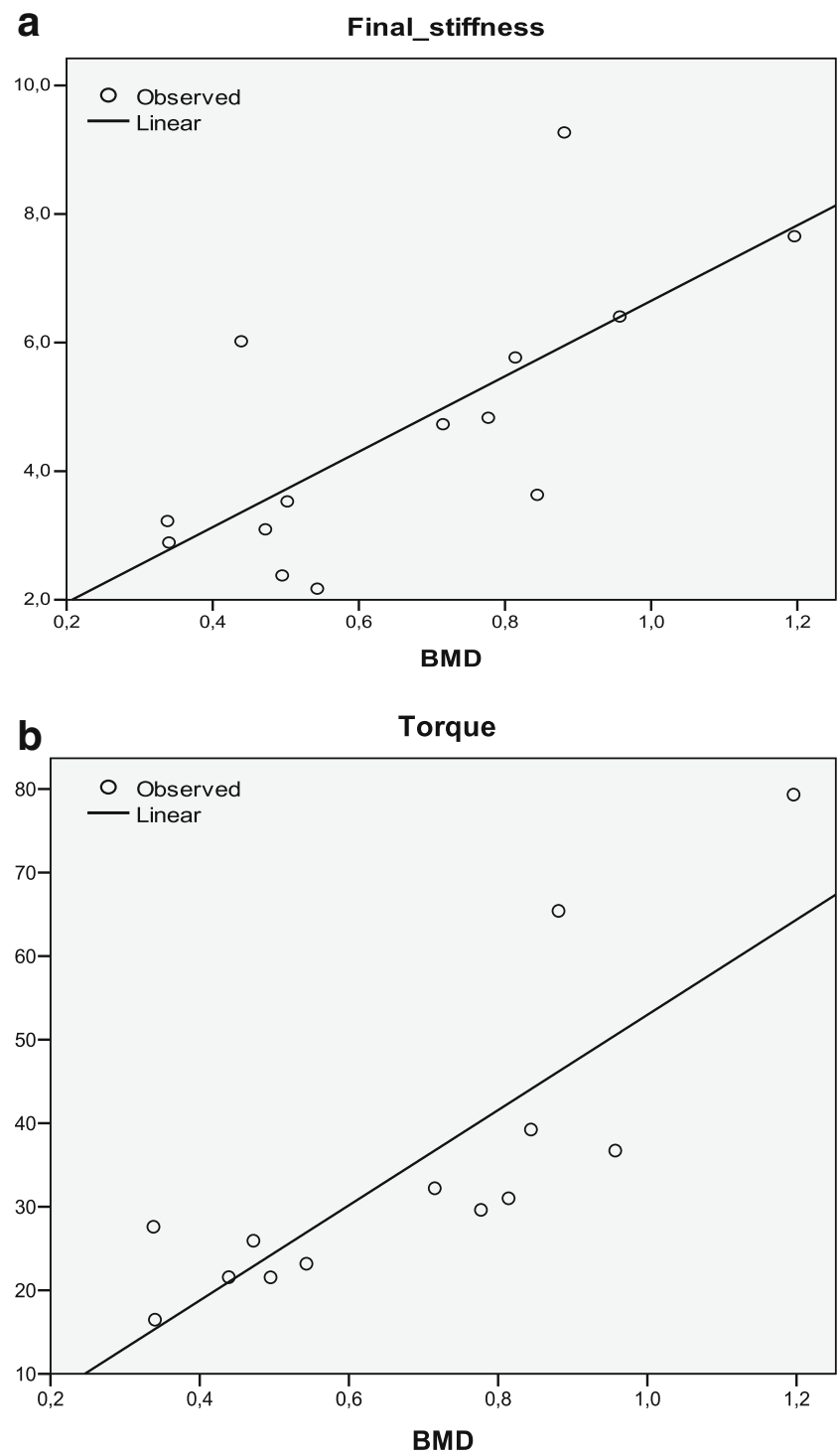

Fig. 5 a Regression analysis with curve estimation $(B M D=$ dependent, final stiffness=independent, $\beta=0.71, p=0.004)$. b Regression analysis with curve estimation $(\mathrm{BMD}=$ dependent, torque $=$ independent, $\beta=0.83$, $p=0.001$ )

chanical study $[1,2]$. Differences between long-stem and standard components did not show differences with respect to torsional stiffness or torque to failure of the bone. The initial fixation was comparable between the specimens, concluded from the initial torsional stiffness. However, failure to fatigue stress is an issue, as can be concluded form the study results. An important factor related to this is the BMD: the lower the BMD, the higher the likelihood of failure to torque. All components moved progressively in the bone-cement interface during the cyclic loading tests, starting at the level of the humeral condyles and propagating to the tip of the prosthesis.

The bone-cement interface is often the weakest link in the prosthetic fixation, as can be clinically observed 
during revision surgery for failed elbow components. Inserting a long-stem component for primary surgery will compromise the bone stock more proximally in the humeral metaphysis. Consequently, revision surgery in the presence of long-stem prostheses is often associated with more severe bone loss compared to the smaller metaphyseal components. This implies that revision surgery necessitates an extended long-stem component, sometimes combined with bone impaction grafting [14]. For these reasons and the fact that both standard and longstem components fail at their prosthesis tip, we favour inserting standard components for primary surgery if possible. In cases of inadequate bone stock around the condyles, a long-stem humeral component could be chosen. Furthermore, final stiffness in the standard components was higher compared to the long-stem components.

A limitation of the study was the small sample size; due to limited availability of cadaveric bone, it was difficult to match enough relatively oversized and undersized components to the humerus.

Our study showed that the torsional stiffness and torque to failure remain constant when inserting relatively small components ('low ratio'). Inserting a relatively large component did not enhance the torsional stiffness and the torque to failure in this study; on the contrary, there might be a negative effect of inserting a relatively large component with a high bone to prosthesis ratio.

All fractures during the single torsion tests started at the condylar ridges. The condylar ridges sustain the condyles and excavating them too much may weaken these pillars. Progression of fractures from condyles to the component's proximal tip, as was found in this study, is a reason for using 'short-stem' standard components in order to limit the size of the fracture and preserve bone support for future revision surgery.

Acknowledgements We would like to thank the Department of Medical Instrumentation for building the custom-made testing device and the Department of Anatomy and Embryology for their assistance during insertion of the prostheses. Both departments are affiliated to the Leiden University Medical Center.

We received financial support from the Annafonds for building the custom-made testing device.

Conflict of interest There is no conflict of interest.
Open Access This article is distributed under the terms of the Creative Commons Attribution Noncommercial License which permits any noncommercial use, distribution, and reproduction in any medium, provided the original author(s) and source are credited.

\section{References}

1. Trail LA, Nuttall D, Stanley JK (2002) Comparison of survivorship between standard and long-stem Souter-Strathclyde total elbow arthroplasty. J Shoulder Elbow Surg 11:373-376

2. Ikävalko M, Belt EA, Kautiainen H, Lehto MU (2002) Revisions for aseptic loosening in Souter-Strathclyde elbow arthroplasty: incidence of revisions of different components used in 522 consecutive cases. Acta Orthop Scand 73:257-263

3. Ikävalko M, Lehto MU, Repo A, Kautiainen H, Hämäläinen M (2002) The Souter-Strathclyde elbow arthroplasty. A clinical and radiological study of 525 consecutive cases. J Bone Joint Surg Br 84:77-82

4. van der Lugt JC, Geskus RB, Rozing PM (2004) Primary SouterStrathclyde total elbow prosthesis in rheumatoid arthritis. J Bone Joint Surg Am 86:465-473

5. van der Lugt JC, Rozing PM (2004) Systematic review of primary total elbow prostheses used for the rheumatoid elbow. Clin Rheumatol 23(4):291-298

6. Schneeberger AG, King GJ, Song SW, O’Driscoll SW, Morrey BF, An KN (2000) Kinematics and laxity of the Souter-Strathclyde total elbow prosthesis. J Shoulder Elbow Surg 9:127-134

7. Valstar ER, Garling EH, Rozing PM (2002) Micromotion of the Souter-Strathclyde total elbow prosthesis in patients with rheumatoid arthritis. 21 elbows followed for 2 years. Acta Orthop Scand 73(3):264-272

8. van der Lugt JC, Geskus RB, Rozing PM (2005) Limited influence of prosthetic position on aseptic loosening of elbow replacements: 125 elbows followed for an average period of 5.6 years. Acta Orthop 76(5):654-661

9. Souter WA (1990) Surgery of the rheumatoid elbow. Ann Rheum Dis 49(Suppl 2):871-882

10. Pöll RG (1994) Souter-Strathclyde total elbow arthroplasty. A prospective clinical study and a biomechanical investigation. Thesis, Leiden University Medical Center, The Netherlands

11. Blum J, Machemer H, Baumgart F, Schlegel U, Wahl D, Rommens PM (1999) Biomechanical comparison of bending and torsional properties in retrograde intramedullary nailing of humeral shaft fractures. J Orthop Trauma 13(5):344-350

12. Stoffel K, Cunneen S, Morgan R, Nicholls R, Stachowiak G (2008) Comparative stability of perpendicular versus parallel double-locking plating systems in osteoporotic comminuted distal humerus fractures. J Orthop Res 26(6):778-784

13. Shibuya K, Hagino H, Morio Y, Teshima R (2002) Cross-sectional and longitudinal study of osteoporosis in patients with rheumatoid arthritis. Clin Rheumatol 21(2):150-158

14. Loebenberg MI, Adams R, O'Driscoll SW, Morrey BF (2005) Impaction grafting in revision total elbow arthroplasty. J Bone Joint Surg Am 87(1):99-106 